\title{
A Rare Cause of Supraventricular Ectopic Beats and Angina in a Young Patient
}

\author{
Monica Jurcău ${ }^{1,2}$, Mariana Floria ${ }^{1,2}$, Bogdan Mazilu ${ }^{1,2}$, Anca Ouatu ${ }^{1,2}$, Daniela Maria Tanase ${ }^{1,2}$ \\ 1 "Sf. Spiridon" Clinical Emergency Hospital, Iaşi, Romania \\ 2 "Grigore T. Popa" University of Medicine and Pharmacy, Iaşi, Romania
}

\section{ABSTRACT}

Introduction: We present the case of a young man with multiple atherosclerotic risk factors and a rare cause of angina and supraventricular ectopic beats. Case presentation: A 35-year-old man with one-year history of stable angina pectoris and supraventricular ectopic beats, without any medication, presented to the emergency room complaining of anterior chest pain that occurs at moderate physical exertion. The rest electrocardiogram, transthoracic echocardiography, and chest X-ray were normal; the exertion stress test was also normal, except for supraventricular ectopic beats (trigeminal rhythm). Due to the intermediate pre-test probability of coronary artery disease, he was evaluated using coronary computed tomography angiography. An anomalous origin of the right coronary artery from the left coronary sinus, with an inter-arterial course and without any atheroma plaques, was observed. During hospitalization the evolution was stable, without complications. The patient was further referred to a cardiac surgery clinic to evaluate the possibility of surgical treatment of this anomaly. Conclusions: Coronary artery anomalies are very rare; however, they present multiple implications in current practice. The most severe complication of this condition is represented by sudden death in young patients due to malignant ventricular arrhythmias. Imaging diagnostic techniques allow for a rapid, noninvasive diagnosis of this rare cause of angina.

Keywords: anomalous coronary origin, right coronary artery, inter-arterial course

\section{ARTICLE HISTORY}

Received: May 2, 2018

Accepted: June 5, 2018

\section{INTRODUCTION}

Coronary artery anomalies affect less than $1 \%$ of the general population. ${ }^{1}$ These anomalies are the second most frequent cause of sudden cardiac death in young athletes. ${ }^{2}$ Anomalies of the right coronary artery emerging from the opposite coronary sinus are much more common and were described for the first time in 1948 by White and Edwards. ${ }^{3}$ These can follow different trajectories: pre-pulmonic, retro-aortic, inter-arterial, transseptal (subpulmonic), and retro-cardiac. ${ }^{1}$ The prevalence of typical angina and that of major adverse cardiac events were reported as significantly higher in patients with a high inter-arterial course than in those with a low inter-arterial course. ${ }^{4}$ In these patients, the resting electrocardiogram is often normal, and evidence of stress-induced ischemia is not always present. ${ }^{1}$ Clinically, the symptoms can vary according to the course of the coronary artery, including angina pectoris, syncope, palpitations, dyspnea, arrhythmias, cardiomyopathy, myocardial infarction, and sudden death, even in the ab- 
sence of atherosclerosis, but most often it is a silent condition. ${ }^{3,5}$ The anomalies with an inter-arterial course are also classified into two subtypes according to the location of the anomalous right coronary artery ostium: high inter-arterial course (between the aorta and the pulmonary artery) and low inter-arterial course (between the aorta and the right ventricular outflow tract)..$^{4}$ Coronary computed tomography (CT) angiography is a noninvasive method for diagnosing such malformations, being able to visualize not only the coronary ostium abnormality, but also the arterial course and the distal extremities of the artery. ${ }^{6}$

\section{CASE REPORT}

A 35-year-old man was admitted in our hospital through the emergency room, after presenting for anterior chest pain of 5-10 minutes duration, which occured at moderate physical exertion, without irradiation, accompanied by dyspnea, palpitations, sweating, and physical asthenia. The symptoms presented an insidious onset 1 year prior to his presentation, with progressive accentuation in the last months. The patient had been diagnosed with dyslipidemia one year previously, for which he was not under any medication. He also presented a significant family history of cardiovascular disease, his father dying at 60 years of acute myocardial infarction.

On physical examination, we found a body mass index of $31.48 \mathrm{~kg} / \mathrm{m}^{2}$ (class I obesity); cardiac examination revealed frequent premature heart sounds followed by a post-extrasystolic pause, a blood pressure of $140 / 85 \mathrm{mmHg}$, and a peripheral pulse of $72 \mathrm{bpm}$. The respiratory, digestive, genitourinary, and neurologic examination were without pathological changes.

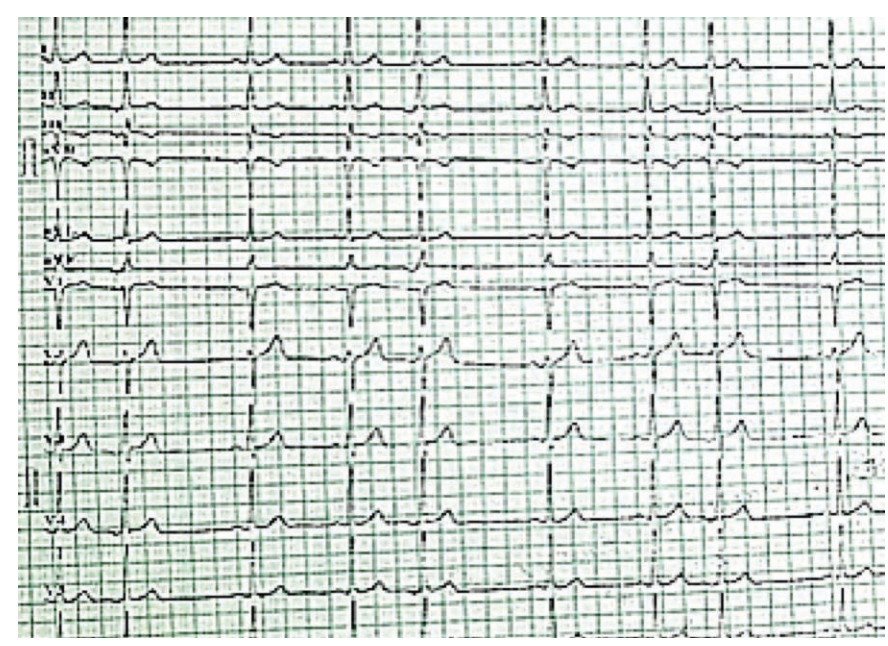

FIGURE 1. The 12-lead resting electrocardiogram at admission showing atrial trigeminal rhythm
The 12-lead resting electrocardiogram at admission showed sinus rhythm, intermediate electrical axis, and persistent atrial trigeminal rhythm (Figure 1). The ergometric stress test revealed the appearance of atrial bigeminal rhythm after the first 3 minutes (Figure 2).

The complete blood count and biochemical profile were normal, including MB creatine kinase ( $9 \mathrm{U} / \mathrm{L}$ ), but showed dyslipidemia (total cholesterol $236 \mathrm{mg} / \mathrm{dL}$; low-density lipoproteins - LDL $173 \mathrm{mg} / \mathrm{dL}$; and high-density lipoproteins - HDL $38 \mathrm{mg} / \mathrm{dL}$ ). The patient had a normal chest X-ray.

Abdominal ultrasound examination pictured normal size liver with homogeneous echogenicity and hepatic steatosis. The gallbladder, pancreas, spleen, and kidneys were normal. The echocardiographic assessment did not record any pathological changes; there was a good global and regional left ventricular function, with a left ventricular ejection fraction of $55 \%$ and no valvular or pericardial abnormalities.

Due to the intermediate pre-test probability of coronary artery disease, the patient was evaluated using coronary CT angiography. This examination revealed that the right coronary artery (RCA) originated from the left side wall of the aorta, approximately $8 \mathrm{~mm}$ cranial to the sino-tubular junction, the proximal segment having an inter-arterial route (between the aorta, the trunk of the pulmonary artery, and the right ventricular ejection tract), without atheromatous plaques or detectable stenoses (Figure 3 and Figure 4). There was a normal CT appearance for the rest of the coronary arteries, the pericardium, the trunk and major branches of the pulmonary arteries, as well as the thoracic aorta. There were no calcified atheromatous plaques in the coronary arteries.

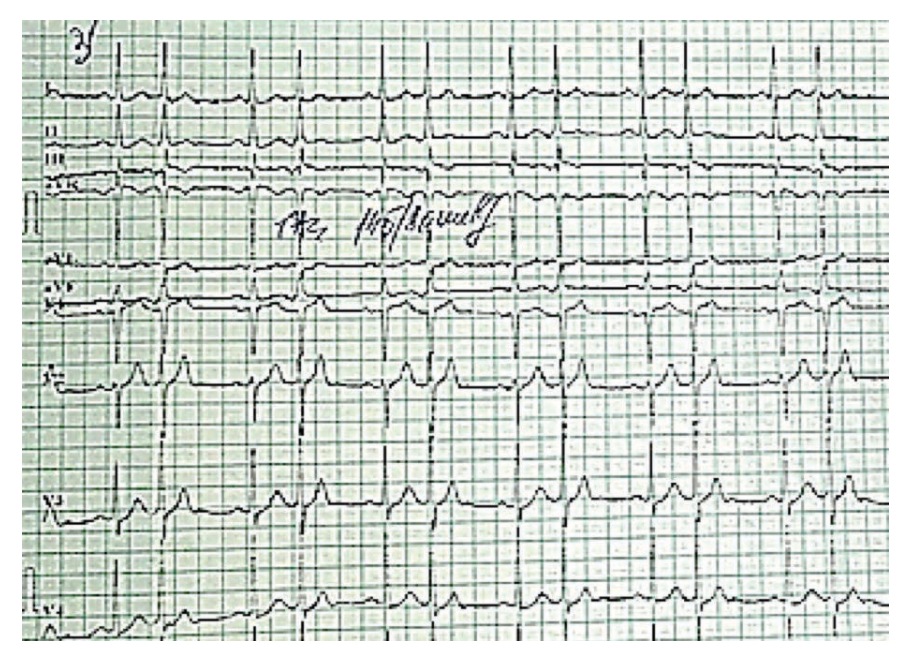

FIGURE 2. The electrocardiogram during ergometric stress test showing atrial bigeminal rhythm 


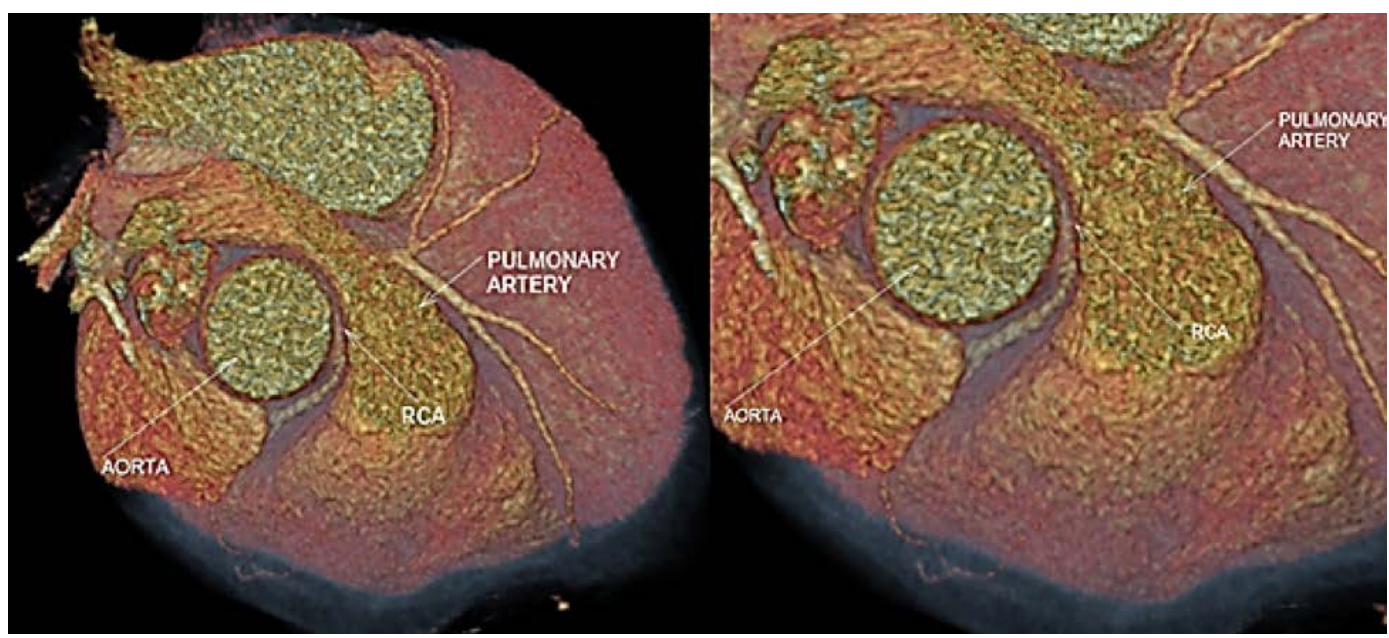

FIGURE 3. 3D reconstruction of a CT image showing the anomalous origin of the right coronary artery (RCA)

During hospitalization, the patient was treated with beta-blockers, antiplatelets, statins, and metabolic medication, with significant improvement in symptomatology.

The patient was discharged after 7 days of hospitalization without complications and was further referred to a cardiac surgery clinic to evaluate the possibility of surgical or interventional treatment (stenting) of the congenital malformation.

The publication of the case has been authorized by the patient, as well as by the hospital where he was referred to.

\section{DISCUSSIONS}

Anomalous origin of the coronary artery from the left coronary sinus is classified as a benign but potentially serious anomaly. ${ }^{7}$ The presence of an inter-arterial tract of this artery is associated with a more severe prognosis and an increased risk of sudden death, explained by the fact that physical effort determines the expansion of the root of the aorta and the pulmonary trunk, which can increase the angulation of the coronary artery and diminish the lumen, thus limiting the blood flow. ${ }^{1,8}$

One out of 3 cases of sudden death in the young population is caused by abnormalities of the coronary arteries, especially those with intramural and inter-arterial trajectory, which underlines the importance of an early diagnosis of this condition. ${ }^{9}$ Coronary abnormalities are often found incidentally in middle-aged to elderly patients. ${ }^{2}$ Anomalies located at the level of coronary ostium are able to promote, through their own physiopathological mechanisms, myocardial ischemia and heart failure. This could be caused by their aberrant anatomical course in different territories, which can lead to compression and angulation, thus causing blood flow disruption. ${ }^{8,10}$

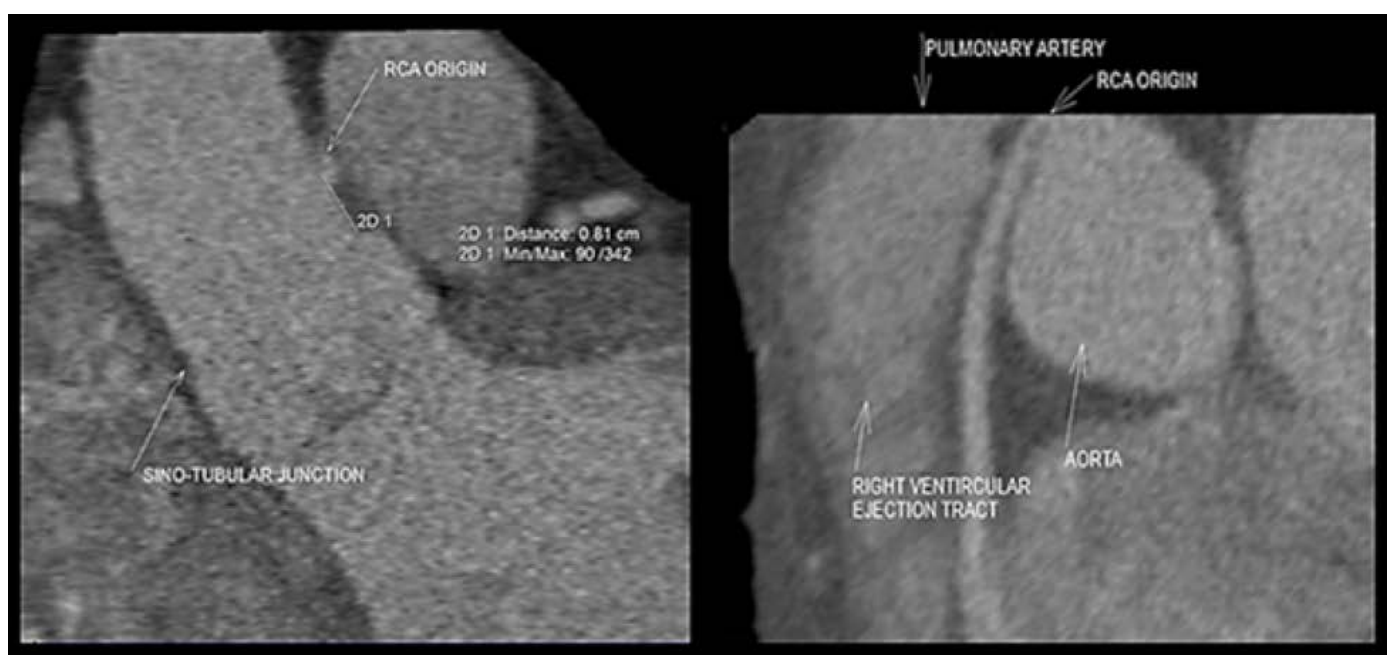

FIGURE 4. CT images showing the anomalous origin of the right coronary artery (RCA) 
The fact that the coronary anomaly of the patient was not associated with atheromatous plaques or stenoses could indicate a favorable short-term prognosis; however, angina pectoris, myocardial infarction, syncope, or ventricular tachycardia have been found in patients with coronary artery anomalies even in the absence of any coronary atherosclerosis. ${ }^{9-11}$ In the long term and in the presence of obesity and sleep apnea, these patients are exposed to a higher risk of sudden death. ${ }^{12}$

Data from the literature shows that there might be a link between supraventricular arrhythmia and the right coronary artery, because in $50-60 \%$ of cases, the origin of the sinus node artery is in the proximal portion of the right coronary artery. ${ }^{1}$ In this clinical case, during the electrocardiogram exercise test, atrial ectopic beats became much more frequent.

One of the surgical techniques quoted in the literature, used with good prognosis in similar cases, consists in the creation of a bypass between the right internal mammary artery and the right coronary artery, creating a new ostium in the left coronary sinus. Other suggested techniques are represented by: (1) patch angioplasty using pulmonary artery wall tissue; (2) stenting the proximal intramural portion of the right coronary artery by percutaneous coronary intervention; (3) increasing the distance between the common pulmonary artery and the aorta by translocating the pulmonary artery root and mobilizing its bifurcation; (4) a surgical approach using the right gastroepiploic artery as a coronary bypass graft. ${ }^{9}$ However, during the stenting of the proximal intramural portion of the right coronary artery by percutaneous coronary intervention, a marked coronary angulation could represent a risk factor for severe complications. ${ }^{13}$

Coronary CT angiography is currently considered the golden standard method for diagnosing coronary artery anomalies, offering good spatial resolution based on image contrast. ${ }^{11}$ In addition, this examination is very important for choosing the best treatment: surgical or interventional, whenever this could be feasible.

\section{CONCLUSIONS}

The presented case highlights the need for an increased attention in the emergency unit to cases that seem simple at first sight, but which can have a potential fatal outcome. An early diagnosis in these cases could be crucial for patient survival. Recognition of this disease in young patients with unexplained angina, essential for timely initiation of an appropriate treatment, could be significantly improved by the use of modern imaging techniques such as computed tomographic angiography.

\section{CONFLICT OF INTEREST}

Nothing to declare.

\section{REFERENCES}

1. Villa A, Sammut A, Nair A. Coronary artery anomalies overview: The normal and the abnormal. World J Radiol. 2016;8:537555. doi: 10.4329/wjr.v8.i6.537.

2. Rossel L, Bonnier H, Sonck J. Anomalous right coronary artery in a middle-aged patient. Medicine. 2016;95:49(e550). doi: 10.1097/MD.0000000000005508.

3. Ho J, Strickman N. Anomalous Origin of the Right Coronary Artery from the Left Coronary Sinus. Tex Heart Inst J. 2002;29:37-39.

4. Lee JH, Hong JY, Kim YH, et al. Anomalous origin of the right coronary artery from the left coronary sinus with an interarterial course: subtypes and clinical importance. Radiology. 2012;262:101-108. doi:10.1148/radiol.11110823.

5. Keswani AN, Dann K, Ramee S. Anomalous right coronary artery: a case report. Ochsner J. 2014;14:287-291.

6. Kim Y, Seo B, Do K-H, et al. Coronary artery anomalies: Classification and ECG-gated Multi-Detector Row CT Findings with Angiographic Correlation. Radiographics. 2006;26:317333. doi: 10.1148/rg.262055068.

7. Wang SS, Chen JM, Zeng GH, et al. The coronary artery sandwich anomaly. Int J Cardiol. 2014;172:e196-e198. doi: 10.1016/j.ijcard.2013.12.074.

8. Lee BY. Anomalous Right Coronary Artery From the Left Coronary Sinus With an Interarterial Course: Is It Really Dangerous? Korean Circ J. 2009;39:175-179. doi: 10.4070/ kcj.2009.39.5.175.

9. Fuglsang S, Heiberg J, Byg J, et al. Anomalous origin of the right coronary artery with an interarterial course and intramural part. Int J Surg Case Rep. 2015;14:92-94. doi: 10.1016/j.ijscr.2015.07.018.

10. Greet B, Quinones A, Srichai M, et al. Anomalous Right Coronary Artery and Sudden Cardiac Death. Circ Arrhythm Electrophysiol. 2012;5:e111-e112. doi: 10.1161/ CIRCEP.112.978635.

11. Renchao G, Saini A, Ahmed I, et al. Interarterial course of anomalous right coronary artery: Pathophysiology, diagnosis and treatment. Radiol Case Rep. 2017;12:664-667. doi: 10.1016/j.radcr.2017.06.006.

12. Salaru DL, Plesoianu CE, Olaru A, Georgescu Arsenescu C. Obstructive Sleep Apnea and Cardiovascular Events: Clinical Correlations. Rev Chim (Bucharest). 2017;68:248-250.

13. Macovei LG, Presura RM, Anghel L, et al. Coronary stent entrapment. Postepy Kardiol Interwencyjnej. 2014;10:216-218. doi: 10.5114/pwki.2014.45154. 